\title{
Dark Matter Halos and the Main Sequence of Starforming Galaxies
}

\author{
Hervé Aussel $^{1}$, Sébastien Peirani ${ }^{2}$ and Laurent Vigroux ${ }^{1,2}$ \\ ${ }^{1}$ Laboratoire AIM Paris-Saclay \\ CE-Saclay, Bat 709, 91919 Gif-sur-Yvette, France \\ email: herve.aussel@cea.fr \\ ${ }^{2}$ Institut d'Astrophysique de Paris \\ 98 bis boulevard Arago, 75014 Paris, France
}

\begin{abstract}
We investigate why hydrodynamical numerical simulations have difficulties (Weinmann et al. 2011) in reproducing the Main Sequence (MS) of star-forming galaxies, i.e. the fact that galaxies forming stars lie on a tight power law sequence in the stellar mass $\left(M^{*}\right)$, star formation rate (SFR) plane (Schreiber et al. 2015). Instead of trying to improve the agreement of simulations with the observations by modifying the subgrid recipes of baryons, we take here a step back to check whether the accretion onto dark matter halos is consistent with the existence of the main sequence of star forming galaxies.
\end{abstract}

Keywords. galaxies: evolution, dark matter

\section{Method}

We use a large high resolution N-body dark-matter only simulation performed with GADGET-2 (Springel 2005) for a $\Lambda$ CDM universe using Planck Collaboration (2013) cosmology. We track the baryon accretion by tagging the DM particules on their first crossing of the virial radius of each halo, and counting them only once, to simulate the fact that baryons are deposited in the DM halo. Our simulations thus allow us to compare the accretion of baryons onto dark matter halos as function of halo mass and redshift. In order to compare with observations, we convert halo masses to stellar masses using the measured relation of Behroozi et al. (2010), and we can directly check whether our accretion rates are compatible with the SFR observed at a given stellar mass for a given redshift.

\section{Results}

At all redshifts, we find that the baryon accretion at the virial radius follows a power law with a slope close to 1.05. The distribution of accretion rates is well described by a double lognormal distribution recalling of the structure of the MS and Starburst in Sargent et al. (2012) models. Baryon accretion at $R_{v i r}$ is always larger than the SFR in the central galaxy hosted by the halo, However, the amount of accreted gas is not enough to sustain SF if the SF efficiency is of the order of few percent as observed locally.

\section{References}

Behroozi, P., Conroy, C., \& Wechsler, R. H., 2010, ApJ 717, 379

Planck Collaboration, 2013 A\&A 571, 16

Sargent, M. T., Béthermin, M., Daddi, E. \& Elbaz, D. 2012 ApJ 747, L31

Schreiber, C., Pannella, M., Elbaz, D., Béthermin, M., Imani, H. et al. 2015, A\&A, 575, 74

\&Springel, V., 2005, MNRAS, 364, 1105

Weinmann, S. M., Neistein, E. \& Dekel, A. MNRAS, 417, 2737 